\title{
Homeostasis en la ciencia escolar: análisis del tema y resultados de una propuesta para su trabajo en el aula
}

\section{Homeostasis in school science: analysis of the subject and results of a proposal for classroom work}

\section{Liliana Esther Mayoral Nouveliére ${ }^{(1)}$, Francisco González García ${ }^{(2)}$ y José Antonio Naranjo Rodríguez ${ }^{(2)}$}

(1) Universidad Nacional de Cuyo, Mendoza, República Argentina (2) Departamento de Didáctica de las Ciencias Experimentales, Universidad de Granada

Resumen: El trabajo presenta un breve repaso del nacimiento del concepto de homeostasis en la historia de la fisiología. Se ha realizado un estudio de aula con estudiantes de tercer curso de secundaria apreciando las dificultades que estos presentan para construir una visión holista del concepto de homeostasis. La ciencia escolar porta una iconicidad múltiple, derivada del lenguaje experto, que en muchos casos se revela como un obstáculo para el aprendizaje de los estudiantes novatos. Siendo la homeostasis un concepto básico en la estructura del curriculum nacional se hace una llamada para ahondar en su investigación didáctica.

Palabras clave: Enseñanza de la Biología, Homeostasis, Regulación, Curriculum, Iconos.

Abstract: The paper shows a brief overview of the origin of the concept of homeostasis in Physiology. We have conducted a study of classroom with high school students appreciating the difficulties that these presented to build a vision of the concept of homeostasis holistic. School science has multiple iconicity, derived from the expert language, which in many cases is revealed to be an obstacle for the novice student learning. Being the homeostasis a basic concept in the structure of the national curriculum, we made a call for research in this topic.

Keywords: Biology teaching, Regulation, Homeostasis, Curriculum, Icons,

(Fecha de recepción: junio, 2015, y de aceptación: abril, 2016)

DOI: 10.7203/DCES.30.6319 


\section{Introducción: la compleja construcción del concepto de homeostasis. Desde la disciplina biológica a la escuela}

La construcción del concepto de homeostasis es uno de los mejores ejemplos donde podemos ver las complejas relaciones entre la ciencia erudita disciplinar y la ciencia escolar, atendiendo a su traslación al curriculum escolar, a la visión que ofrecen los textos escolares, y a la comprensión que resuelven los estudiantes en el trabajo en el aula. Nos encontramos con un concepto que podemos denominar como concepto estructurante, en la denominación de Gagliardi (1986), y donde el papel de la historia de la disciplina juega un rol esencial (Gagliardi y Giordan, 1986).

El concepto de homeostasis en la ciencia disciplinar tiene como figura clave a Claude Bernard, quien en su obra "Introducción a la medicina experimental" (1865) plantea una epistemología reflexiva, fuertemente pragmática en fundamentos biológicos aplicados a la medicina y alejada de concepciones vitalistas propias de la medicina antigua (López Piñero, 2000). Bernard rompe con viejos conceptos y pone los fundamentos del estudio científico de la vida sobre el análisis experimental de los fenómenos fisiológicos en relación con procesos físico-químicos. Expone la noción de complejidad en base a una serie de fenómenos simples que se jerarquizan estructuralmente para cumplir un objetivo esencial: la regulación de la constancia del medio interno. Bernard comunica sus trabajos con un lenguaje que permite construir imágenes y/o modelos diversos; anclados a veces en antiguas representaciones, como cuando utiliza la analogía de una serpiente que se muerde la cola significando el concepto de vida (Bernard, 1865). El objeto de estudio, la regulación interna de los seres vivos, fue descrito a lo largo de la historia con lenguajes muy diversos; se nombró, explicó y describió con muy distintos recursos para construir modelos mentales igualmente variados.

Estructuras como la hipófisis, de gran importancia homeostática, ya había sido descrita en la Grecia antigua pero sin vinculación funcional. En el siglo XVII Lower explica que los productos hipofisarios son segregados a la sangre, aunque desconocía que estos eran estímulos de otras funciones. Las dudas funcionales y relacionales superaban a los conocimientos certeros. En 1889, Brown-Sèquard (discípulo de Claude Bernard) registra los primeros enunciados explicando la función vinculante de las secreciones de la hipófisis sobre el funcionamiento de otras estructuras. El término lingüístico que servía para identificar estas secreciones, lo enuncian Bayliss y Starling en 1905, para designar la función de la secretina. Estas sustancias excitantes que provocaban un movimiento al interactuar con otras, se denominaron hormonas. Este vocablo asumió otras denominaciones genéricas anteriores para diversas sustancias químicas y desterró las concepciones antiguas de sutiles sustancias o espíritus animales (Henao, 
2004; Mason, 1996). Las hipótesis y las justificaciones de Bernard y discípulos comenzaron a adquirir nombre propio, aun cuando la histología estaba en construcción, e incluso la teoría celular, enunciada en 1839, empezaba a asentarse en el campo de la Biología.

El término homeostasis derivado del griego y que etimológicamente significa "estabilidad semejante o similar" $($ Homeo $=$ similar; stasis $=$ estado; estabilidad), fue seleccionado por Walter Cannon en 1926 para dar identidad lingüística a las explicaciones pretéritas de Claude Bernard. Los sucesos de investigación científica simultáneos y posteriores contribuyeron a ampliar el número de fenómenos singulares y de fenómenos vinculantes que se involucran bajo tal denominación. Entre esos fenómenos que ampliaron el campo de la homeostasis es muy destacable la conexión neuronal entre hipotálamo y neurohipófisis. Los puentes vinculantes entre el sistema hormonal y el nervioso se registraron de la mano de Gregorio Marañón en 1915 (Argente et al., 2003) quien otorgó significado biológico a las secreciones internas como resultado de sus reflexiones en torno al análisis de casos patológicos. Los trabajos de Bargmann y Scharrer, en 1950 (Purpura, 1998) fortalecieron la unidad hipotálamo-hipófisis al denominar "células nerviosas glandulares" a aquellas observadas en el tálamo segregando "gotitas" del mismo modo que las células endocrinas.

Jacobi (1990) resalta que todo este proceso de construcción histórica de la disciplina científica está plagada de construcciones, reconstrucciones y mutaciones de términos y conceptos que correlacionan con hipótesis y esquemas mentales que deben ser transmitidos desde la comunidad científica a la comunidad escolar.

En este proceso, la noción general de homeostasis se rige como un concepto estructurante de la Biología y posee una propiedad esencial de los mismos en la Biología: el hecho que sus propiedades macroscópicas solo son explicables desde lo microscópico. Este hecho hace que en muchas ocasiones la Biología sea particularmente difícil de aprender (Gagliardi, 1986).

La herramienta didáctica más utilizada en el aula sigue siendo el manual o libro de texto, aún con el avance acelerado de las nuevas tecnologías de la información, en particular en contextos escolares donde su implantación es aún reducida. En los manuales escolares de Biología, temáticas complejas suelen ilustrarse con dibujos o gráficos utilizando el pretendido potencial didáctico de las imágenes (Pérez de Eulate y Llorente, 1998). Sin embargo estas representaciones icónicas y su particular semiótica (verbal e icónica) tiene discontinuidades y dificultades para el alumnado y en ocasiones no es totalmente adecuada para los fines didácticos que se les presupone (Perales, 2006; Maldonado et al., 2007).

En el estudio de los materiales curriculares por excelencia, los libros de texto editados en la República Argentina, encontramos en la iconografía una 
constante aunque con dos periodos distintos (Mayoral et al., 2011). Los libros de textos escolares desde 1945 hasta la actualidad muestran que el tratamiento de la homeostasis se centra básicamente en las estructuras anatómicas. Los análisis de textos anteriores a 1990 sostienen los conceptos fisiológicos con iconografía muy limitada, con proposiciones y narraciones sencillas y con la ausencia del tratamiento del sistema inmune. Las producciones posteriores a 1990 , por contra, presentan abundancia de iconografía, en general a pleno color. La iconografía de dibujos figurativos y de fotografías son los más frecuentes para la función de regulación y control en el organismo humano. Por ejemplo para el concepto de hormona los dibujos figurativos y las fotografías son lo más común y muy escasos son los dibujos con signos normalizados. Esta ausencia o muy escasa presencia de esquemas o diagramas de fisiología relacional, provocan descripciones muy incompletas de los procesos fisiológicos (Mayoral et al., 2011).

En el trabajo que desarrollamos a continuación, tras esta breve revisión histórica, presentamos un ejemplo de trabajo en el aula con estudiantes de educación secundaria argentinos, incidiendo en la importancia de las representaciones, las denominaciones, en definitiva de la semiótica. Entendemos que para un correcto aprendizaje de los conceptos básicos de homeostasis y regulación interna hay que apostar por unas representaciones icónicas sencillas pero adecuadas y una demanda de resolución de problemas para los estudiantes.

\section{Metodología}

\section{El Curriculum escolar}

Alfabetizar científicamente es una demanda en el ámbito de la educación para ciudadanos del siglo XXI. En la República Argentina los diseños curriculares son diseñados por los gobiernos provinciales a partir de las bases emanadas por el Ministerio de Educación de la Nación (Ministerio de Educación, 2006a). La homeostasis aparece como un concepto básico en el desarrollo del curriculum de ciencias naturales y en el desarrollo de diversos cursos de la educación secundaria. El tratamiento curricular de la homeostasis, según los diseños vigentes en la República Argentina, se aplica en los cursos entre $1^{\circ}$ año y $4^{\circ}$ año de secundaria, lo cual implica, estudiantes cuyas edades oscilan entre los 13 y 16 años.

La expectativa de logro que se declara en el curriculum es "aproximarse a la concepción de organismo humano como sistema abierto y complejo a través del reconocimiento de las interrelaciones de los diferentes sistemas que lo componen y utilizar este conocimiento para adoptar actitudes que contribuyan al cuidado de la salud", así como alcanzar una capacidad que permita "el reconocimiento de la función de relación en el organismo humano a través del análisis de situaciones donde se evidencien procesos de captación y procesamien- 
to de la información y elaboración de respuestas que permitan avanzar en la construcción de la noción del organismo humano como un sistema integrado, abierto y complejo (Ministerio de Educación, 2006b) . En el desarrollo del curriculum durante la educación secundaria se espera que "la estructura conceptual sobre homeostasis construida por los estudiantes de educación secundaria sepa integrar conceptos básicos (denominados nodos), tales como "fuente de secreción", "acción a distancia", "molécula y "noción de red". Estos nodos son importantes para la comprensión del funcionamiento de la homeostasis en el organismo vivo global, y como tal lo contempla la construcción en la ciencia erudita (Rumelhard, 1998; Curtis et al., 2008; Purves et al., 2002).

Nuestra hipótesis de partida al indagar en el aula sobre estos conocimientos, teóricamente adquiridos, es que el conocimiento sobre homeostasis construido por los estudiantes de educación secundaria obligatoria se sitúa dentro de un paradigma mecanicista, lo cual podemos concretar en que hay un escaso logro al resolver tareas de comprensión de representaciones icónicas y lingüísticas habituales en sus textos de estudio ligados a problemas de salud donde la homeostasis se encuentra íntimamente presente. Como segunda hipótesis planteamos que se puede lograr una mejora en estos logros si se plantean una secuencia de actividades planificada para el trabajo con la semiótica de los nodos y trabajando temas de salud adecuados.
Participantes, e instrumentos utilizados

La población participante donde se realizó el estudio fue un grupo de 50 estudiantes de $3^{\circ}$ año de secundaria (o primer curso de polimodal, con edades entre 15 y 16 años), de la escuela 4-001 "Dr. J. V. Zapata" de la Ciudad de Mendoza, Argentina.

Atendiendo al curriculum vigente, los estudiantes de tercer año habían abordado en su totalidad los contenidos del objeto de estudio, la noción de homeostasis en el ciclo lectivo anterior. A todos los participantes se les evaluó mediante un pre-test. Posteriormente se aplicó una secuencia didáctica completa a una submuestra de 19 estudiantes, seleccionados al azar de entre los cincuenta iniciales y se les aplicó el postest de evaluación.

Los instrumentos de evaluación y de trabajo en la secuencia del aula se resumen en el Cuadro I.

\section{Aplicación}

Se diseñó y aplicó, inicialmente, un instrumento con escala tipo Likert sobre ideas, conceptos y capacidades en torno a la regulación y el control. Resuelta la instancia de pre-test, se utilizaron cuatro instrumentos portando iconografía frecuente en la presentación temática de los libros de ciencia escolar, acompañados con preguntas abiertas y textos acordes a los íconos.

La finalidad fue la de observar el grado de resolución a partir de la inter- 


\section{Cuadro I \\ Tipo de instrumentos aplicados en el trabajo}

\begin{tabular}{|c|c|}
\hline Instrumento de evaluación & Función \\
\hline $\begin{array}{l}\text { - Instrumento con escala tipo Likert (Pre y post- } \\
\text { test). } \\
\text { El test se diseñó con } 32 \text { ítems, quedando } \\
\text { finalmente configurado con un total de } 26 \text { (con } \\
\text { un coeficiente de Cronbach de } 0.885 \text {; Mayoral } \\
\text { et al., 2011). } \\
\text { Noción de red } 10 \text { ítems, noción molécula } 5 \\
\text { ítems, noción fuente de secreción } 4 \text { ítems, } \\
\text { noción acción distancia } 7 \text { ítems. }\end{array}$ & $\begin{array}{l}\text { Detección de ideas, conocimientos y capacidades } \\
\text { sobre el concepto de homeostasis }\end{array}$ \\
\hline \multicolumn{2}{|c|}{$\begin{array}{l}\text { Algunos ejemplos de ítems: } \\
\text { Valora el grado de acuerdo o desacuerdo que muestras con los siguientes enunciados, siendo } 1 \\
\text { (totalmente en desacuerdo) y } 6 \text { (totalmente de acuerdo): } \\
\text { - La homeostasis es una función compleja que depende solo del sistema hormonal } \\
\text { - La homeostasis es una función compleja que involucra al sistema nerviosos relacionado con el } \\
\text { sistema endocrino } \\
\text { - Interpreto el concepto de molécula como una estructura química que puede estimular o inhibir el } \\
\text { funcionamiento de una célula } \\
\text { - Las hormonas son sustancias que son transportadas a los diferentes lugares del organismo por } \\
\text { medio de la circulación sanguínea } \\
\text { - Las hormonas son sustancias que pueden ser transportadas por el líquido extracelular } \\
\text { - Interpreto que las funciones que involucran a la homeostasis se desarrollan generando un vínculo } \\
\text { de red móvil }\end{array}$} \\
\hline Secuencia didáctica aplicada & Función \\
\hline $\begin{array}{l}\text { - Instrumentos de respuesta abierta con } \\
\text { iconografía inserta } \\
\text { - Instrumentos con iconografía inserta y con } \\
\text { espacio para resolver iconografía propia. } \\
\text { - Instrumentos con iconografía inserta y con } \\
\text { demanda de elaboración y aplicación de } \\
\text { etiquetas } \\
\text { - Resolución de problemas en torno al nivel de } \\
\text { glucemia. }\end{array}$ & $\begin{array}{l}\text { Test aplicados a la sub-muestra poblacional. } \\
\text { Trabajo en el aula sobre la comprensión de } \\
\text { la semiótica tradicional y funcional sobre el } \\
\text { concepto de homeostasis. } \\
\text { Indagar sobre la capacidad de interpretación y } \\
\text { de resolución de problemas en torno al concepto } \\
\text { estructurante. }\end{array}$ \\
\hline
\end{tabular}

pretación iconográfica y de relación con el lenguaje verbal que acompaña a las ilustraciones o con la necesidad de nombrar a partir de la comprensión de una representación, colocando etiquetas verbales. Los instrumentos constituyeron una guía o secuencia didáctica, resueltos por la sub-muestra poblacio- nal. La idea de un último instrumento de evaluación referido a resolución de problemas, fue la de revelar la capacidad de aplicar los conceptos centrales en diferentes situaciones de regulación interna. Finalmente el post-test, nos permitió recoger la interacción de los estudiantes en su totalidad con las cues- 
tiones planteadas y apreciar su progreso en la construcción de la regulación del medio interno de los seres vivos.

La secuencia didáctica trabaja esencialmente con la lingüística y la iconicidad, siendo estos recursos didácticos considerados fuertemente. La búsqueda de vocablos comunes para la enunciación verbal y de imágenes (íconos) y de secciones ampliadas o extraídas de estas imágenes (hipoíconos), atendiendo a diferentes conceptos que han emergido a lo largo de la historia de la fisiología, se constituyeron en el nexo didáctico esencial para que la noción estructurante homeostática fuera emergiendo en los estudiantes. El hilo conductor se estableció sobre la regulación de glucemia desde el nivel pancreático hasta la función hipotalámica, asociando funciones del Sistema Nervioso y estableciendo puentes con las funciones inmunitarias.

\section{Resultados y Discusión}

La aplicación de los instrumentos y el tratamiento de las respuestas al pre y post-test permitieron extraer información que se puede estructurar en base a los nodos fuentes que se eligieron. El test, diseñado como una escala Likert, obliga a los estudiantes a emitir su acuerdo o desacuerdo (en una escala de 6 valores) sobre una serie de enunciados que permiten detectar y valorar sus ideas y conocimientos. Consideramos que cuando muestra su total acuerdo/total desacuerdo y de acuerdo/ en desacuerdo, según el enunciado, hay una idea correcta y conocimiento del enunciado.

El Cuadro II muestra una síntesis básica de los resultados. Siendo conscientes de la limitación de la muestra poblacional, creemos que un análisis detallado de los estadísticos de los diferentes ítems que referencian a cada nodo podría oscurecer el significado global del estudio y, por ello, mostramos una comparación sobre la conceptualización media global de los estudiantes.

En el pre-test se aprecia que solo 1 de cada tres o cuatro estudiantes muestra una comprensión adecuada (23$35 \%)$. Resaltar que en la sub-muestra a la que aplicamos la secuencia didáctica $(n=19)$, los porcentajes eran iguales salvo para el nodo "distancia", pero dentro de los valores generales.

Tras el trabajo didáctico aplicado en la secuencia con el grupo de 19 estudiantes, podemos ver que en todos los nodos hay variaciones favorables; en el caso de la noción de fuente de secreción es menor y mayor la noción de distancia. En todo caso, la permanencia de las ideas anteriores está fuertemente conservada. Diferentes trabajos muestran que el concepto de homeostasis y/o regulación neuroendocrina son conceptualizados por los estudiantes con gran dificultad (Lazaroweitz y Penso, 1992; Bahar et al., 1999; Michael, 2007).

Más allá de este escaso avance, acorde con la idea histórica de la dificultad en la construcción de los conceptos nodales de la homeostasis, nos resulta de gran importancia el trabajo con el subgrupo de la muestra poblacional 


\section{Cuadro II.}

Datos comparativos sobre porcentajes de estudiantes con respuestas que indican una correcta comprensión de los nodos básicos en el concepto de homeostasis (porcentajes son la media para los diferentes ítems

\begin{tabular}{|l|c|c|c|}
\hline Conceptos básicos o nodos & Pre-test (n=50) & Pre-test (n=19) & Post-test (n=19) \\
\hline Fuente de secreción (4 ítems) & $\mathbf{3 1 \%}$ & $\mathbf{3 1 \%}$ & $\mathbf{3 6 \%}$ \\
\hline Distancia (7 ítems) & $\mathbf{2 3 \%}$ & $\mathbf{3 2} \%$ & $\mathbf{5 0 \%}$ \\
\hline Molécula (5 ítems) & $\mathbf{3 1 \%}$ & $\mathbf{3 1 \%}$ & $\mathbf{4 4 \%}$ \\
\hline Noción de red (10 ítems) & $\mathbf{3 5 \%}$ & $\mathbf{3 5 \%}$ & $\mathbf{4 2 \%}$ \\
\hline
\end{tabular}

$(\mathrm{n}=19)$ que permitió extraer algunas señales respecto de la continuidad de ideas o los cambios conceptuales resueltos por los estudiantes.

En el Cuadro III se muestran algunos ejemplos de la iconografía utilizada durante la secuencia didáctica planteada a los estudiantes. El trabajo didáctico comenzó demandando la resolución a partir de la interpretación de íconos de tipo dibujo figurativo tradicionales o canónicos (tipo 1). El reconocimiento de la estructura representada se produjo en 18 de los 19 estudiantes; diez de ellos reconocen que la flecha relacional señala parte de esa estructura, pero tan solo 2 estudiantes vinculan correctamente el hipoícono. En el caso de la representación tradicional del cerebro, la identificación la realizaron 14 estudiantes, pero el hipoícono de la hipófisis no fue identificado en ningún caso.

Cuando se trabajó con dibujos figurativos (tipo 2) que debían completarse según las ideas propias, tres de cada cuatro estudiantes expresaba ideas correctas pero solo uno de cada cinco era capaz de resolver la representación icónica de una molécula en la vía comunicante. En algunos casos no se resolvía ningún tipo de estos dibujos figurativos. Tres de cada cinco estudiantes resolvieron de forma adecuada y elaboraron un texto coherente para la tarea tipo 3 . Dos estudiantes no pudieron elaborar un texto propio.

Indagar respecto del significado otorgado por los estudiantes a diversos íconos y ciertas palabras es una necesidad y se requieren análisis sobre diversos tipos de documentos e iconografías. Es muy probable que los estudiantes tengan referencias erróneas que pueden provenir de su trayecto escolar o a partir de las mismas referencias que marcan los libros de textos escolares. Emerge la necesidad de estudiar el desajuste en la comunicación verbal y visual, es decir, los desajustes semióticos que los estudiantes manifiestan.

Planteado al finalizar el desarrollo de la secuencia didáctica una cuestión sobre el nivel de la glucemia, con dibujos y signos esquemáticos (tipo 4), se advirtió que permanecía un porcentaje de los estudiantes sin poder expresar 


\section{Cuadro III}

Ejemplos de iconografía utilizada en la secuencia didáctica de aula

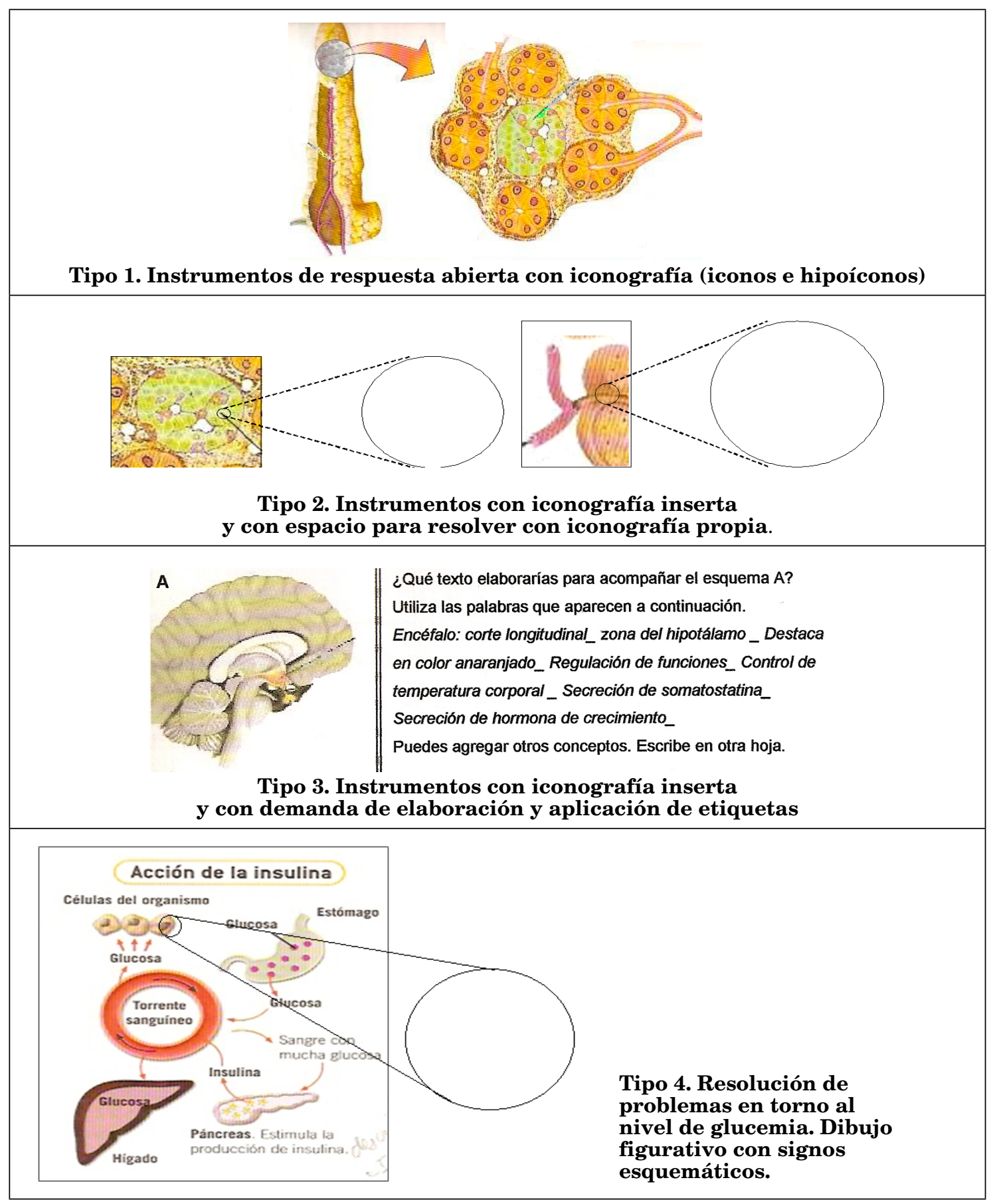


claramente asociación entre algunos conceptos. Uno de cada tres presentaba graves problemas de resolución de iconos propios y dos de cada tres aún mantenían diversos errores; en un solo caso la resolución era plena. Cada construcción resuelta plantea singularidades, casi a modo de un mapa mental, pero en general con pocos nexos verbales. Sin embargo la solución al problema general de regulación fue resuelta adecuadamente por cuatro de cada cinco estudiantes.

En síntesis, del trabajo de resolución del grupo de estudiantes, apreciamos dos principales obstáculos. Primero que la homeostasis aparece fuertemente ligada al sistema endocrino, con sinonimia al hormonal y poco vinculado a cualquier función del sistema nervioso u otros elementos del organismo. Segundo, que la pluralidad de nombres y denominaciones de las células, moléculas segregadas, vías de comunicación y secreción, etc, produce una evidente confusión incluso entre estructuras orgánicas simples e impide una visión holística de los sistemas orgánicos.

Por otra parte en el campo de la representación icónica es necesario señalar que el ícono es un medio para el acceso al saber y, para que la lectura de una imagen científica no sea arbitraria existe un código basado sobre convenciones, donde la unidad gráfica y la unidad pertinente del sistema semiótico dependen de la codificación previa de una experiencia perceptiva. En la Biología, en general, y en esta temática en particular no existen con- venciones gráficas y semióticas que a fuerza de su multiplicación y repetitividad cobren significación única no ambigua. La notación icónica requiere de su constante explicitación para orientar al lector en su significación. Este hecho fue repetidamente comprobado en la actividad de aula.

Los íconos seleccionados e insertos para construir una unidad didáctica (dentro de un libro de texto o no), en definitiva no deben determinar cortes que nieguen la realidad, ni provocar una visión reduccionista de aquella. La construcción de un puente entre la visión holista, objetivo a conseguir por la enseñanza, y la visión parcial acotada de los estudiantes debe ser facilitada por la lectura de todos los lenguajes (escrito, íconos y símbolos); sin embargo apreciamos que muchas veces la semiótica construida lo impide. Las llamadas de atención que Kramer (1998) y Chartier (1999) realizan sobre esta problemática escolar se ejemplarizan en el caso de la enseñanza y aprendizaje de la biología, en concreto en el campo de la fisiología.

\section{Conclusiones}

La comprensión de la fisiología en el nivel de enseñanza media discurre entre una multitud de términos y conceptos (Nordquist, 2008), así como de múltiples representaciones icónicas en textos y otros recursos tecnológicos para la enseñanza (Stavrianeas et al., 2008), sin embargo no es un sujeto muy habitual de la investigación educativa. 
Los mecanismos de homeostasis quizás sean uno de los mejores ejemplos para entender que los seres vivos no son una simple suma de partes sino que se necesita una profunda interacción entre constituyentes, sin embargo los materiales curriculares no los presentan de este modo (Cañal, 2008).

Nuestra muestra de estudio es ciertamente muy reducida y las conclusiones deben enmarcarse en tal contexto. Tanto en el grupo inicial como en el subgrupo donde se aplicó una secuencia de trabajo específica apreciamos que la construcción del concepto de homeostasis resulta particularmente difícil, de acuerdo con nuestras expectativas iniciales. En ocasiones encontramos problemas similares al desarrollo histórico de la fisiología.

La comprensión de los mecanismos de regulación interna requieren una fuerte reestructuración de las estructuras cognitivas de los estudiantes. El resultado con el subgrupo en donde se aplicó una secuencia concreta con palabras e íconos comunes a este campo, la semiótica general de la homeostasis, promovió una interpretación más correcta de los procesos de regulación físico-química de los seres vivos, si bien sus resultados fueron modestos, pero confirmamos que es posible una mejora aún en una temática tan compleja.

La presentación de estrategias didácticas que trabajen con problemas reales puede ser de utilidad aunque se requiere de más trabajos en el aula y una mayor atención por parte de la disciplina y especialistas de la didáctica de las Ciencias Biológicas, en particular en los niveles de enseñanza media. Desde el sentido que se apunta por el National Research Council (1996), los modelos de explicación que sobre el concepto estructurante de homeostasis presentan los estudiantes parecen ser fuertemente personales y variables y tenían cierto carácter predictivo cuando se trabajaba con los problemas reales, por lo que sería de interés profundizar en su estudio en diferentes niveles de enseñanza, tanto en su evolución como en niveles de complejidad.

Nos resulta algo sorprendente que siendo la homeostasis un concepto esencial para la comprensión del ser vivo, sin embargo tenga tan poca atención en la investigación didáctica de la disciplina, e incluso su planteamiento en los textos se reduzca a temas muy repetidos. Existe un campo amplio a indagar: desde las propias dificultades de los estudiantes a las posibles secuencias de enseñanza y estrategias para superarlas.

\section{Referencias}

ARGENTE, J.; POZO, J. y CASANUEVA, F. (2003). Hipotálamo e hipófisis. Revisión de la Obra Médica de Gregorio Marañón. Madrid: Fundación Gregorio Marañón.

BAHAR, M.; JOHNSTONE,A.H. y HANSELL,M.H.(1999).Revisitinglearning difficulties in biology. Journal of Biological Education, n ${ }^{\circ} 33(3), 84-86$. DOI: $10.1080 / 00219266.1999 .9655648$ 
BERNARD, C. (1865). Introduction à l'étude de la médecine expérimentale. Cadre de la collection : "Les classiques des sciences sociales ", développée en collaboration avec la Bibliothèque Paul-Émile Boulet. Université du Québec à Chicoutimi. Recuperado de <http://www.uqac. uquebec.ca/zone30/Classiques des sciences sociales/index.html >.

CAÑAL, P. (2008). El cuerpo humano: una perspectiva sistémica. Alambique, Didáctica de las Ciencias Experimentales, $\mathrm{n}^{\circ}$ 58, 8-22.

CHARTIER, R. (1999). La cultura escrita en la perspectiva de larga duración. Cultura escrita, literatura e historia. México: Fondo de la Cultura Económica.

CURTIS, H; BARNES, N; SCHNEK, A y MASSARINI, A. (2008). Biología. $7^{a}$ Edición. Buenos Aires: Editorial Médica Panamericana.

GAGLIARDI, R. (1986). Los conceptos estructurales en el aprendizaje por investigación. Enseñanza de las Ciencias, $\mathrm{n}^{\circ} 4(1)$, 30-35.

GAGLIARDI, R. y GIORDAN, A. (1986). La Historia de las Ciencias: una herramienta para la enseñanza. Enseñanza de las Ciencias, $\mathrm{n}^{\circ}$ 4(3), 253-258.

HENAO, G. (2004). Endocrinología y Epistemología. Revista Colombiana de Obstetricia y Ginecología, n ${ }^{\circ} 55$ (3), 167-179.

JACOBI, D. (1990). Quelques tendances ou effets de figurabilité dans la divulgation des théories immunologiques. Aster, $\mathrm{n}^{\circ}$ 10, 129-143.
KRAMER, S. (1998). Lectura y escritura de maestros. Revista Propuesta Educativa, $\mathrm{n}^{\circ} 19$.

LAZAROWITZ, R. y PENSO, S. (1992). High school students difficulties in learning biology concepts. Journal of Biological Education, $\mathrm{n}^{\circ} 26$ (3), 215-223. DOI: 10.1080/00219266.1992.9655276

LÓPEZ PIÑERO, J. (2000). Cajal. Madrid: Debate-Pensamiento.

MALDONADO, F.; GONZÁLEZ, F. y JIMÉNEZ, M.P. (2007). Las ilustraciones de los ciclos biogeoquímicos del carbono y nitrógeno en los textos de secundaria. Revista Eureka sobre Enseñanza y Divulgación de las Ciencias, $\mathrm{n}^{\circ}$ 4(3) 442-460.

MASON, S. (1996). Historia de la Ciencia. Madrid: Alianza.

MAYORAL, L.; GONZÁLEZ, F. y NARANJO, J.A. (2011). La iconicidad en Biología. construcción del concepto de homeostasis. Saarbrucken. Editorial Académica Española.

MICHAEL, J. (2007). What makes physiology hard for students to learn? Results of a faculty survey. Advances in Physiology Education, ${ }^{\circ}$ 31, 34-40. DOI: 10.1152/advan.00057.2006

MINISTERIO DE EDUCACIÓN, CIENCIA Y TECNOLOGIA DE LA NACIÓN. (2006a). Ley Nacional de Educación, $\mathrm{N}^{\circ}$ 26075. República Argentina

MINISTERIO DE EDUCACIÓN CIENCIA Y TECNOLOGÍA DE LA NACIÓN. (2006b). Núcleos de aprendizaje primario, Tercer Ciclo de Educación Secundaria. Buenos Aires. 
NATIONAL RESEARCH COUNCIL. (1996). National Science Education Standars. Washington DC: National Academy.

NORDQUIST, L. (2008). Physiology education and the linguistic jungle of science. Advances in Physiology Education, $\mathrm{n}^{\circ} 32$, 173-174. DOI: $10.1152 /$ advan.90115.2008

PERALES, F.J. (2006). Uso (y abuso) de la imagen en la enseñanza de las ciencias. Enseñanza de las Ciencias, $\mathrm{n}^{\circ} 24(1), 13-30$.

PÉREZ DE EULATE, L. y LLORENTE, E. (1998). Las imágenes en la enseñanza-aprendizaje de la biología. Alambique, $\mathrm{n}^{\circ}$ 16, 45-53.
PURPURA, D.P. (1998). Berta Scharrer: Biographycal Memoir. Washington D.C.: National Academy Press.

PURVES, W.; SADAVA, D; ORIANS, G y HELLER H. (2002). Vida. La ciencia de la Biología. Madrid: Médica Panamericana

RUMELHARD, G. (1988). Formation, modification et dissolution du concept d'hormone dans l'enseignement. Aster, $\mathrm{n}^{\circ}$ 7, 143-170.

STAVRIANEAS, S.; STEWART, M. y HARMER, P. (2008). Beyond the printed page: physiology education without a textbook? Advances in Physiology Education, $\mathrm{n}^{\circ} 32$, 76-80. DOI: 10.1152/advan.00031.2007 
